\title{
Sulphostin, a Novel Inhibitor of Dipeptidyl Peptidases IV (DPPIV) That Stimulates Hematopoiesis in Mice
}

\author{
Masatoshi Abe, Fuminori Abe, Chihiro Nishimura, Eiji Ichimura, Asuka Ogasawara, \\ Megumi Ichinei, Yasuhiko Muraoka, Tetsushi Saino
}

Received: September 24, 2004 / Accepted: January 7, 2005

(C) Japan Antibiotics Research Association

\begin{abstract}
CD26, a membrane-bound ectopeptidase, is known as an activated $\mathrm{T}$ cell marker with dipeptidyl peptidase IV (DPPIV) activity that has diverse functional roles in the regulation of peptide hormones, neuropeptides, chemokines and growth factors. We recently isolated a novel inhibitor of DPPIV, sulphostin, from culture broth of Streptomyces sp. MK251-43F3. We investigated herein the hematopoietic effect of sulphostin in mice and found that sulphostin induced the production of granulocyte colony-stimulating factor (G-CSF), stimulated myeloblasts in bone marrow, and increased neutrophil numbers in peripheral blood in both normal mice and mice with cyclophosphamide-induced leucopenia. Sulphostin desulfonate, in addition to sulphostin, has a similar inhibitory effect on DPPIV and stimulatory effect on neutrophils. These results suggest that DPPIV/CD26 might be a novel target for hematopoietic stimulation and DPPIV inhibitors including sulphostin and derivatives may be candidates for further development.
\end{abstract}

Keywords sulphostin, dipeptidyl peptidase IV (DPPIV) inhibitor, neutrophil stimulator

\section{Introduction}

Dipeptidyl peptidase IV (DPPIV, E.C.3.4.14.5) is a protease with a homodimer type II membrane protein, which is highly expressed on the surface of endothelial and immune cells and identical to CD26 antigen [1]. The cDNA for human DPPIV/CD26 encodes a protein of 766 amino acids with 9 potential glycosylation sites. DPPIV/CD26 consists of three extracellular regions; a membrane-proximal glycosylated region starting with a 20 -amino acid flexible stalk region, a cysteine-rich region, and a 260- amino acid $\mathrm{C}$-terminal region with DPPIV activity. DPPIV removes $\mathrm{N}$ terminal dipeptides from a number of proteins having either a Pro or Ala residue in the penultimate position. DPPIV/CD26 regulates the activity of many peptide hormones, neuropeptides, cytokines and growth factors via its peptidase function [2]. On the other hand, the surface protein DPPIV/CD26 is capable of mediating cell-matrix and cell-cell contact through interactions with other molecules or proteins probably including collagen, fibronectin, and adenosine deaminase. Recently, it was described as a co-stimulatory molecule in T cell activation and reportedly associated with CD45. DPPIV/CD26 is involved in regulating the proliferation of the same hematopoietic and T cell-lineage, and associated with HIV pathogenesis. Experimental models and clinical studies addressing the role of these enzymes and the effect of specific inhibitors have paved the way to novel therapeutic concepts in immunology [3], oncology [4], diabetes [5] and so on.

Sulphostin was first isolated from the culture broth of Streptomyces sp. MK251-43F3 as a novel inhibitor of
F. Abe (Corresponding author), C. Nishimura, E. Ichimura, A. Ogasawara, M. Ichinei, T. Saino: R. \& D. Division, Nippon Kayaku Co. Ltd., 31-12 Shimo 3-chome, Kita-ku, Tokyo, 1158588 Japan, E-mail: fuminori.abe@nipponkayaku.co.jp
M. Abe, Y. Muraoka: Microbial Chemistry Research Center, 1423 Kamiosaki 3-chome, Shinagawa-ku, Tokyo, 141-0021 Japan 
DPPIV [6]. Here we show that sulphostin has G-CSFinducing activity and increases neutrophil numbers in peripheral blood in mice.

\section{Materials and Methods}

\section{Mice}

Male ICR mice, 7 weeks of age, were supplied from Charles River Japan, Inc., Kanagawa, Japan. They were kept under specific pathogen-free conditions with clean air of our animal facility. The animal care and use were in accordance with institutional guidelines.

\section{Agents}

Sulphostin (configuration of $\mathrm{C}-3$ and $\mathrm{P}$ is $S$ and $R$, respectively), $\mathrm{P}$-epi sulphostin (configuration of $\mathrm{C}-3$ and $\mathrm{P}$ is $S$ and $S$, respectively) and a sulfonic acid group-deficient analogue (sulphostin desulfonate) were synthesized as reported previously [7, 8]. Val-boroPro (PT-100) was synthesized by the method as reported by Coutts et al. [9]. The structures are shown in Fig. 1. The agents were dissolved in saline solution and filtered with a Millipore filter $(0.22 \mu \mathrm{m})$. All agents and experiments contained less than $0.1 \mathrm{ng} / \mathrm{ml}$ of endotoxin as assessed with the Limulus lysate assay.

\section{Measurement of DPPIV Inhibitory Activity}

The in vitro assay for DPPIV inhibitory activity was performed as reported previously [7]. Briefly, 0.1 M Tris (hydroxymethyl)-aminomethane/maleic acid buffer solution (pH 7.2, $100 \mu \mathrm{l}), 3.2 \mathrm{mM}$ Gly-Pro- $\beta$-naphthylamide $(25 \mu \mathrm{l}$, Bachem, Switzerland), and an aqueous solution (50 $\mu \mathrm{l})$ of the agents were added into 96-well micro plates. The resultant solution was warmed for 10 minutes at $37^{\circ} \mathrm{C}$, and then the DPPIV solution $(25 \mu \mathrm{l})$ was added to the solution. The combined solution was allowed to react for 1 hour at $37^{\circ} \mathrm{C}$. The reaction was terminated by adding a solution (100 $\mu \mathrm{l}$ ) of $0.2 \%$ Fast Garnet GBC salt (Sigma, U.S.A.) in $0.5 \mathrm{M}$ sodium citrate buffer solution ( $\mathrm{pH} 3.78$ ) including $10 \%$ polyoxyethylene sorbitan monolaurate (Wako, Japan). The absorbance at $525 \mathrm{~nm}$ was measured.

\section{Sulphostin Treatment}

ICR mice were intravenously administered saline or sulphostin daily as indicated. In the leukopenic model, ICR mice received an intraperitoneal injection of cyclophosphamide (CP) at $200 \mathrm{mg} / \mathrm{kg}$ on day 0 and were intravenously administered saline or sulphostin daily starting on day 1 as indicated in the figure legend.

Peripheral blood was collected from the abdominal or<smiles>N[C@H]1CCCN(P(N)(=O)NS(=O)(=O)O)C1=O</smiles>

sulphostin

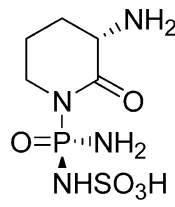

P-epi sulphostin sulphostin desulfonate

Fig. 1 Chemical structures of sulphostin and the derivatives.

orbital vein in the treated mice on a predetermined day for the determination of blood cell counts, changes in segmented neutrophils and cytokine production.

\section{Hematology}

The absolute number of white blood cells was counted using an automatic blood cell counter (MEK-6258, Nihon Kohden Co., Tokyo) and the proportion of segmented neutrophils was determined in blood smears with the Wright Stain (Sigma).

\section{Cytokine ELISA}

Levels of G-CSF, IFN $\gamma$ and IL12 were determined by quantikine enzyme linked immunosorbent assay (ELISA) according to the attached product information (R\&D Systems, Minneapolis, MN).

\section{Histopathological Examination}

The tissues including bone marrow, spleen, thymus and liver were embedded in paraffin, sectioned and stained with hematoxylin with eosin. Histopathological observations were conducted.

\section{Statistics}

The student $t$ test and parametric Williams test were calculated using Microsoft Excel software (Richmond, WA).

\section{Results}

\section{Inhibition of DPPIV Activity by Sulphostin and Related Compounds}

Sulphostin and the related desulfonate strongly inhibit DPPIV activity similar to that by Val-boroPro (PT-100). The $50 \%$ inhibitory concentrations $\left(\mathrm{IC}_{50}\right)$ of sulphostin and the related desulfonate were 21 and $94 \mathrm{nM}$, respectively, although P-epi sulphostin did not show any inhibitory activity (Table 1). 


\section{Increase in the Absolute Number of Neutrophils by Sulphostin}

The effect of sulphostin on hematopoiesis was explored in mice, when sulphostin was intravenously administered to ICR mice at a dose of $2 \mathrm{mg} / \mathrm{kg}$ to $250 \mathrm{mg} / \mathrm{kg}$ for 5 consecutive days (Fig. 2). Sulphostin dose-dependently increased the absolute number of white blood cells in peripheral blood and the increase was significant at a dose over $10 \mathrm{mg} / \mathrm{kg}$ of sulphostin. There were no decreases of body weight or abnormal clinical signs in mice observed following sulphostin administration even at $250 \mathrm{mg} / \mathrm{kg}$. All mice were autopsied 24 hours after the last administration. Spleen weights had also doubled in sulphostin-treated mice in comparison with those in saline-treated mice. When sulphostin was administered at doses of 50 or $250 \mathrm{mg} / \mathrm{kg}$ (data not shown), liver weights were increased about $20 \%$ in comparison with those in saline-treated mice. These results suggest that sulphostin stimulated hematopoiesis not only in bone marrow but also in the spleen and liver.

Table 1 Inhibition of DPPIV activity by sulphostin and the derivatives

\begin{tabular}{lc}
\hline \multicolumn{1}{c}{ Agent } & $\mathrm{IC}_{50}(\mathrm{nM})$ \\
\hline Sulphostin & 21 \\
P-epi sulphostin & $>1000$ \\
Sulphostin desulfonate & 94 \\
Val-boroPro & 7.4
\end{tabular}

DPPIV activity was measured by degradation of Gly-Pro- $\beta$ naphthylamine as reported previously [7].

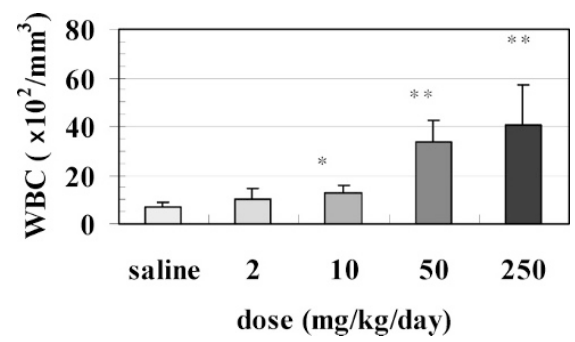

Fig. 2 Absolute number of white blood cells in mice after multiple treatments with sulphostin. Sulphostin was intravenously administered to ICR mice once a day for 5 consecutive days. Absolute number of white blood cells in peripheral blood from the abdominal vein was counted 24 hours after the last administration. Data expressed as the mean \pm S.D. of $3 \sim 4$ mice/group. ${ }^{*} p<0.05,{ }^{*} p<0.01$ as compared to saline control by parametric Williams $t$-test.

\section{Cytokine Production and Histopathological Examination}

To examine the precise stimulation of hematopoiesis, sulphostin was intravenously administered at a dose of $30 \mathrm{mg} / \mathrm{kg}$ for three days and the absolute number of segmented neutrophils and level of cytokines were measured. As shown in Fig. 3A, the absolute number of segmented neutrophils began to increase 12 hours after the third administration of sulphostin and peaked 24 hours after the administration. Coincidently, the G-CSF concentration in blood significantly increased 24 hours after the third administration (Fig. 3B). Although sulphostin stimulated IFN $\gamma$ production, the increase was not significant. There

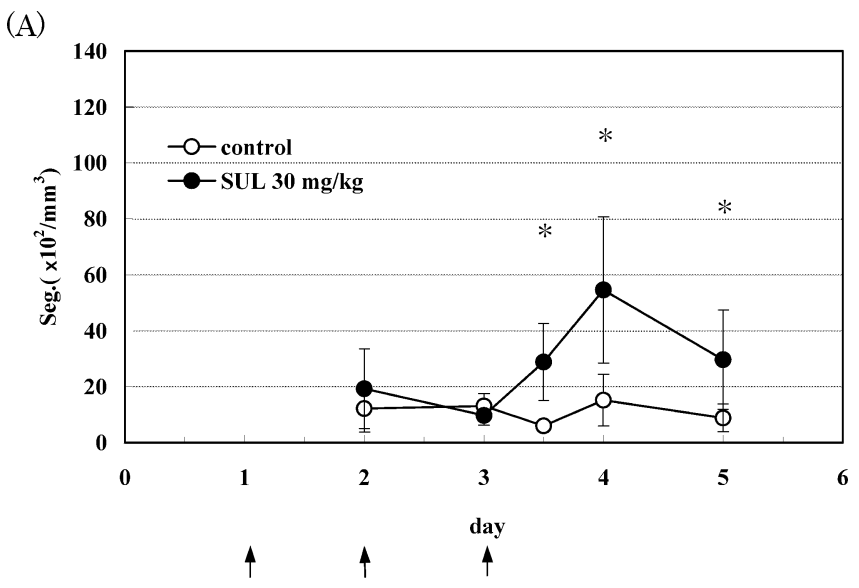

(B)

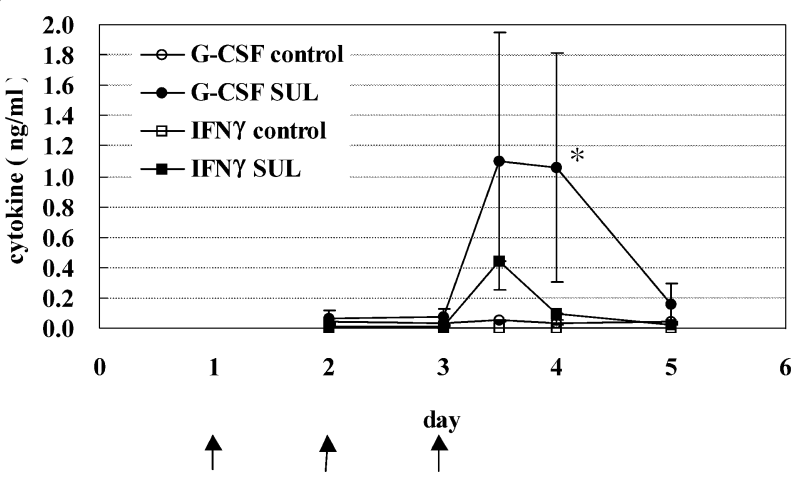

Fig. 3 Effect of sulphostin on absolute number of segmented neutrophils and production of G-CSF and IFN $\gamma$ in mice. Sulphostin (SUL, $30 \mathrm{mg} / \mathrm{kg}$ ) was intravenously administered to ICR mice once a day for 3 days. Mice were sacrificed 24 hours after the first or second administration and 12, 24, and 48 hours after third administration. (A) Segmented neutrophils in peripheral blood from the orbital vein were determined in blood smears with Wright stain. (B) Concentration of G-CSF and IFN $\gamma$ in serum from the abdominal vein were measured with ELISA. Data expressed as the mean \pm S.D. of 3 mice/group. ${ }^{*} p<0.05$ as compared to saline control with paired Student $t$-test. 
was no stimulation of IL12 production observed in the experiment (data not shown).

Further, the histopathological confirmation was independently conducted under the same schedule of sulphostin administration in Fig. 3 in mice (Table 2). Histopathological examination showed an increase in the number of myeloblasts in bone marrow, an increase in the number of myeloblasts and megakaryocytes in the red pulp of spleen, thinning of the cortex in thymus and an increase in the number of myeloblasts 24 hours after the second and third administrations of sulphostin. Representative specimens of bone marrow are shown in Fig. 4.

\section{Stimulation of bone marrow recovery in cyclophosphamide-induced leucopenia}

Finally the effect of sulphostin and the related desulfonate on hematopoietic recovery following treatment with myelosuppressive dose of cyclophosphamide was examined (Fig. 5). Mice injected with cyclophosphamide at a dose of $200 \mathrm{mg} / \mathrm{kg}$ showed a reduction of segmented neutrophils 4 days after cyclophosphamide injection. Sulphostin and the related desulfonate both at a dose of $30 \mathrm{mg} / \mathrm{kg}$ showed a tendency to stimulate the recovery of hematopoiesis in mice with cyclophosphamide-induced leucopenia. The desulfonate at a dose of $100 \mathrm{mg} / \mathrm{kg}$ showed significant stimulation of recovery. However, P-epi sulphostin did not show any stimulation of bone marrow corresponding to the inhibition of DPPIV (data not shown).

\section{Discussion}

The present study demonstrated that sulphostin, a potent inhibitor of DPPIV, has a stimulating effect on the increase in myeloblasts in bone marrow, absolute number of neutrophils and production of G-CSF in mice. Sulphostin desulfonate inhibits DPPIV activity and increases neutrophil numbers similar to sulphostin. However, P-epi sulphostin shows no enzyme inhibition or stimulation of myelogenesis. As reported previously, sulphostin, originally isolated from the cultured broth of Streptomyces, was synthesized as three possible stereoisomers whose absolute configurations at $\mathrm{C} 3$ and the phosphorus atom were determined to be $S$ and $R$, respectively, while those of P-epi sulphostin are $S$ and $S$, respectively [7]. This result suggests that the configuration of the phosphorus atom is primarily responsible for the inhibitory activity toward DPPIV. Recently we examined the interaction of sulphostin with the catalytic site of DPPIV and found that all the functional groups on the piperidine ring are crucial to the inhibitory effect of sulphostin on DPPIV, and that the sulfonic acid group, which constructs the amino(sulfoamino)phosphinyl group, contributed to the stability of the compounds [8]. Moreover, those functional groups must be adjoined to the piperidine ring for the inhibitory activity. The size of the nitrogen-containing heterocyclic ring including piperidine appeared to scarcely affect the activity. Therefore, the mechanism behind the relationship between DPPIV inhibition and bone marrow stimulation aroused our interest.

Table 2 Histopathological examination of sulphostin on hematopoietic-stimulation in mice

\begin{tabular}{|c|c|c|c|c|c|c|}
\hline \multirow{2}{*}{ Organ Findings } & \multirow{2}{*}{$\begin{array}{c}\text { Administration time; } \\
\text { Hours after administration; }\end{array}$} & \multirow{2}{*}{$\begin{array}{c}\text { day } 1 \\
24 \text { hours }\end{array}$} & \multirow{2}{*}{$\begin{array}{l}\text { day } 1 \sim 2 \\
24 \text { hours }\end{array}$} & \multicolumn{3}{|c|}{ day $1 \sim 3$} \\
\hline & & & & 12 hours & 24 hours & 48 hours \\
\hline \multicolumn{7}{|l|}{ Spleen } \\
\hline \multicolumn{2}{|c|}{ Increase of myeloblasts in red pulp } & $1 / 3$ & $3 / 3$ & $1 / 3$ & $3 / 3$ & $1 / 3$ \\
\hline \multicolumn{2}{|c|}{ Increase of megakaryocytes } & $0 / 3$ & $3 / 3$ & $2 / 3$ & $3 / 3$ & $3 / 3$ \\
\hline \multicolumn{7}{|l|}{ Mesenteric lymph node } \\
\hline \multicolumn{2}{|l|}{ Granuloma } & $1 / 3$ & $1 / 3$ & $1 / 3$ & $3 / 3$ & $3 / 3$ \\
\hline \multicolumn{2}{|l|}{ Infiltration of neutrophils } & $0 / 3$ & $0 / 3$ & $0 / 3$ & $2 / 3$ & $2 / 3$ \\
\hline \multicolumn{7}{|l|}{ Thymus } \\
\hline \multicolumn{2}{|l|}{ Thinning cortex } & $1 / 3$ & $3 / 3$ & $2 / 3$ & $3 / 3$ & $2 / 3$ \\
\hline \multicolumn{7}{|l|}{ Bone marrow } \\
\hline \multicolumn{2}{|l|}{ Increase of myeloblasts } & $0 / 3$ & $3 / 3$ & $2 / 3$ & $3 / 3$ & $1 / 3$ \\
\hline \multicolumn{2}{|l|}{ Increase of neutrophils } & $0 / 3$ & $0 / 3$ & $0 / 3$ & $1 / 3$ & $2 / 3$ \\
\hline
\end{tabular}

Sulphostin $(30 \mathrm{mg} / \mathrm{kg})$ was intravenously administered to ICR mice once a day for 3 consecutive days. Mice were sacrificed at the indicated hours after administration and histopathological study was examined in specimens prepared from sulphostin-treated mice ( $\mathrm{n}=3$ ). 


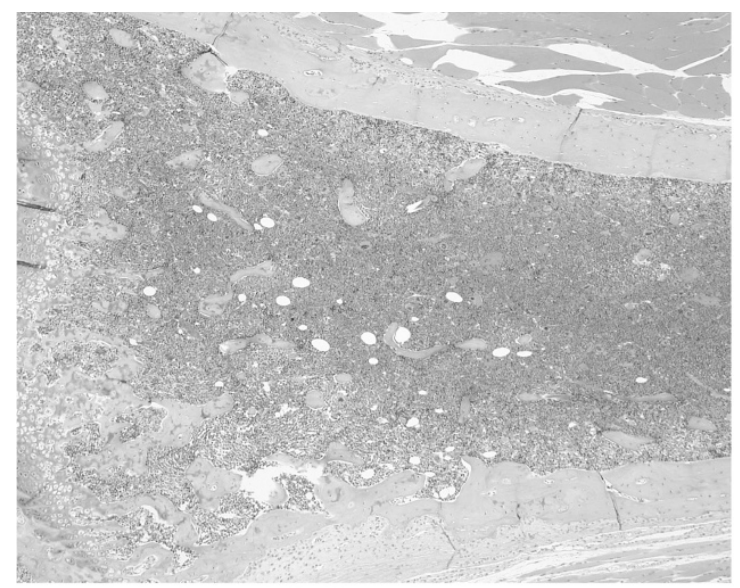

Sulphostin

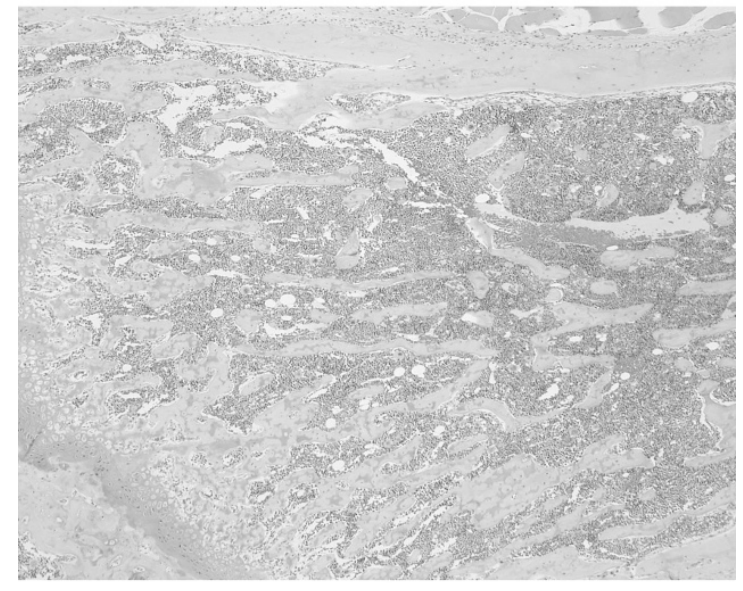

Control

Fig. 4 Changes in the histological morphology of bone marrow in sulphostin-treated mice by HE staining. Representative specimens of bone marrow 24 hours after the third sulphostin $(30 \mathrm{mg} / \mathrm{kg})$ administration (upper) and the control (lower) from the experiment of Table 2, magnification $\times 15$.

CD26/DPPIV (EC 3.4.14.5) is a 110-Kda glycoprotein expressed in various cell types tissues such as renal proximal tubules, intestinal epithelial cells, kidney, liver, placenta and lung. The complete c-DNA and derived amino acid sequence was published in 1992 [10] and the threedimensional crystal structure analysis was determined in 2003 [11]. CD26 is reported to be a marker of autoimmune disease, adenosine deaminase deficiency and HIV pathogenesis. Interestingly, insulin secretion was enhanced and glucose tolerance was improved in mice lacking CD26 [12]. Thus, a variety of DPPIV/CD26 inhibitors have been found and developed for the treatment of type 2 diabetes, as immunosuppressants and hematopoietic stimulators [13]. For instance, Diprotin A (Ile-Pro-Ile), a 1st generation of

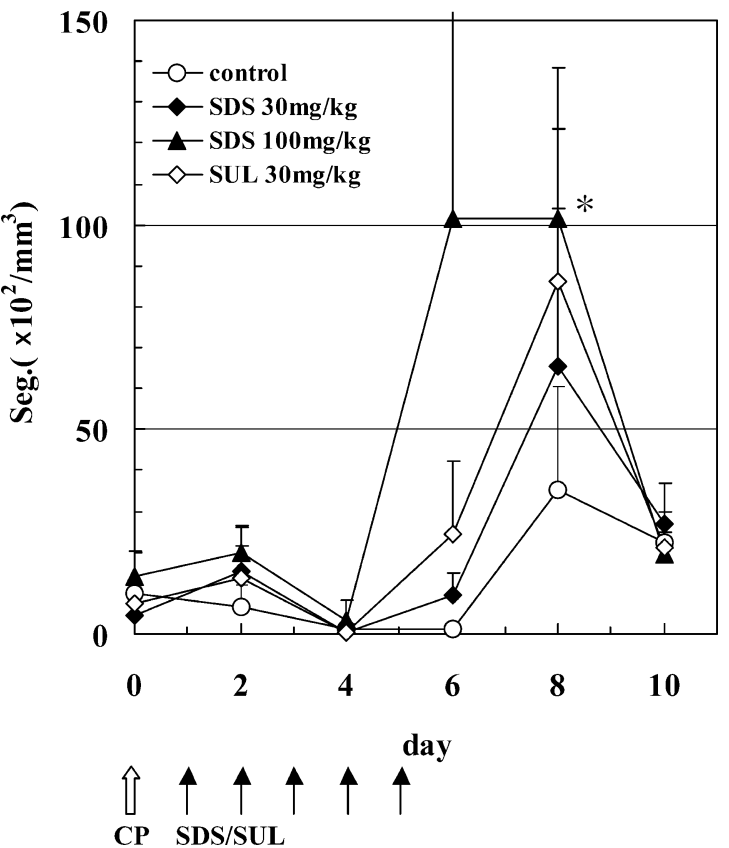

Fig. 5 Effect of sulphostin and the related desulfonate on recovery of segmented neutrophils in mice with cyclophosphamide-induced leukopenia. Leukopenia was induced by intraperitoneal injection of cyclophosphamide (CP) at a dose of $200 \mathrm{mg} / \mathrm{kg}$ on day 0 to ICR mice, and either sulphostin (SUL) or the related desulfonate (SDS) was administered once a day for 5 consecutive days starting from day 1 . Segmented neutrophils in peripheral blood from the orbital vein were determined by standard differential counting. Data expressed as the mean S.D. of $3 \sim 4$ mice/group. ${ }^{*} p<0.05$ as compared to saline control with paired Student $t$-test.

inhibitor [14], Lys[Z(NO2)]-thiazolidide and Lys[Z(NO2)]pyrrolidide showed inhibitory activity against $\mathrm{T}$ cells [15]. LAF237 and MK-0431 showed inhibition of GLP-1 (glucagon-like peptide-1), and are under Phase II clinical trials in patients with type 2 diabetes [16].

CD26/DPPIV truncated various biologically active peptides. Guan et al. observed that the full-length MIP-1 $\beta$ is processed by CD26/DPPIV to the truncated form and that cleavage can be blocked by DPPIV inhibitory peptides derived from HIV Tat or thromboxane A2 receptor [17]. CD26/DPPIV has the ability to cleave the chemokine CXCL12/SDF-1 $\alpha$ (stromal cell derived factor $1 \alpha$ ) at its two prolines. CD26/DPPIV co-distributes and coimmunoprecipitates with CXCR4, a receptor for CXCL12/ SDF-1 $\alpha$, suggesting a functional relationship with SDF-1 [18]. Broxmeyer et al. reported that CXCL12/SDF-1 $\alpha$ chemoattracts hematopoietic stem and progenitor cells (HSC/HPC) and may play a crucial role in the mobilization of $\mathrm{HSC} / \mathrm{HPC}$ from the bone marrow (BM) [3, 19]. 
CD26/DPPIV cleaves dipeptides from the $N$-terminus of polypeptide chains, including CXCL12. CD26 is expressed in normal Sca- ${ }^{+} \mathrm{ckit}^{+}$lin $^{-} \mathrm{HSC} / \mathrm{HPC}$ isolated from mouse $\mathrm{BM}$ and these cells possess CD26 peptidase activity. Recently, it was reported that the inhibition by a DPPIV inhibitor of Diprotin A treatment or genetic deletion of $\mathrm{CD} 26$ in $\mathrm{CD} 26^{-/-}$mice with donor stem cells increased the efficacy of hematopoietic stem cell transplantation about 10 fold. It was hypothesized that CXCL12, which is thought to be involved in the homing of stem cells, was a target of CD26 inhibition which might increase stem cell homing and engraftment [3]. Although G-CSF is not truncated by CD26/DPPIV, other cytokines or chemokines including MIP-1 chemotaxis factor of neutrophils, SDF-1 stimulation factor for hematopoiesis, and others may be involved in the mechanism [12]. CXCR4 is important for mediating the specific migration of bone marrow stromal cells to bone marrow.

On the other hand, Pro-boroPro, well known as a CD26 inhibitor, has similar activity to sulphostin and reportedly stimulates rat granulocyte/macrophage colony formation and thymocyte proliferation [20]. The related analogue of PT-100 (Val-boroPro) showed bone marrow stimulatory activity and anti-tumor activity [21], and a clinical study is ongoing in oncology. However, Jones et al. reported that PT-100 was activated to increase WBC cell numbers in $\mathrm{CD} 26^{-1-}$ knock out mice. Thus, PT-100-associated effects occur via a CD26/DPPIV-independent pathway. They suggested that the hematopoietic target for PT-100 is fibroblast activation protein (FAP) [22]. FAP $\alpha$ is a $95 \mathrm{Kda}$ surface antigen selectively expressed in reactive stromal fibroblasts of epithelial cancer, granulation tissue of healing wounds and malignant cells of bone and soft-tissue sarcoma [23, 24]. FAP $\alpha$ showed $48 \%$ amino acid sequence identity to the CD26/DPPIV. However, CD26 is widely expressed in normal tissues. It is reported that $\operatorname{FAP} \beta$ is identical to CD26, and FAP $\alpha$ and FAP $\beta$ (CD26/DPPIV) closely interact [23]. Further study is needed to clarify the mechanism of bone marrow stimulation by sulphostin and whether the target molecule of sulphostin is CD26/ DPPIV or not.

In summary, sulphostin induces the production of cytokines including G-CSF and stimulates myeloblasts in bone marrow. Our preliminary data indicate that sulphostin and its derivatives have therapeutic potential as a strong inhibitor of DPPIV.

Acknowledgments The authors thank Dr. Takaaki Aoyagi and Dr. Masaaki Ishizuka, Microbial Chemistry Research Center for their helpful advice. The authors also thank Dr. Takumi Yamashita and Ms. Kayo Kikuchi for support and technical assistance.

\section{References}

1. Meester ID, Korom S, Damme JV, Scharpe S. CD26, let it cut or cut it down. Immunol Today 20: 367-375 (1999)

2. Augustyns K, Bal G, Thonus G, Belyaev A, Zhang XM, Bollaert W, Lambeir AM, Durinx C, Goosens F, Haemers A. The unique properties of dipeptidyl-peptidase IV (DPPIV) and the therapeutic potential of DPPIV inhibitors. Current Medical Chem. 6: 311-327 (1999)

3. Christopherson K, Hangoc WG, Mantel CR, Broxmeyer HE. Modulation of hematopoietic stem cell homing and engraftment by CD26. Science 305: 1000-1003 (2004)

4. Cheng JD, Dunbrack RL, Valianou JM, Rogatko A, Alpaugh K, Weiner LM. Promotion of tumor growth by murine fibroblast activation protein, a serine protease, in an animal model. Cancer Res. 62: 67-72 (2002)

5. Villhauer EB, Brinkman JA, Naderi GB, Burkey BF, Dunning BE, Prasad K, Mangold BL, Russell ME, Hughes TE. 1-[[(3-Hydroxy-1-adamantyl)amino] acetyl]-2-cyano$(S)$-pyrrolidine: a potent, selective, and orally bioavailable dipeptidyl peptidase IV inhibitor with antihyperglycemic properties. J. Med. Chem. 46: 2774-2789 (2003)

6. Akiyama T, Abe M, Harada S, Kojima F, Sawa R, Takahashi Y, Naganawa H, Homma Y, Hamada M, Yamaguchi A, Aoyagi T, Muraoka Y, Takeuchi T. Sulphostin, a potent inhibitor for dipeptidyl peptidase IV from Streptomyces sp. MK251-43F3. J Antibiot 54: 744-746 (2001)

7. Abe M, Akiyama T, Nakamura H, Kojima F, Harada S, Muraoka Y. First synthesis and determination of the absolute configuration of sulphostin, a novel inhibitor of dipeptidyl peptidase IV. J Nat Prod 67: 999-1004 (2004)

8. Abe M, Akiyama T, Umezawa Y, Yamamoto K, Nagai M, Yamazaki H, Ichikawa Y, Muraoka Y. Synthesis and biological activity of sulphostin analogues, novel dipeptidyl peptidase IV Inhibitors. Bioorg Med Chem 13: 785-797 (2005)

9. Coutts SJ, Kelly TA, Snow RJ, Kenedy CA, Barton RW, Adams J, Krolikowski DA, Freeman DM, Cambell SJ, Ksiazek IF, Bachovchin WW. Structure-activity relationships of boronic acid inhibitors of dipeptidyl peptidase IV. 1. Variation of the P2 position of Xaa-boroPro dipeptides. J Med Chem 39: 2087 (1996)

10. Darmoul D, Lacasa M, Baricault L, Marguet D, Sapin C, Trotot P, Barbat A, Trugnan G. Dipeptidyl peptidase IV (CD26) gene expression in enterocyte-like colon cancer cell lines HT-29 and Caco-2. Cloning of the complete human coding sequence and changes of dipeptidyl peptidase IV mRNA levels during cell differentiation. J Biol Chem 267: 4824-4833 (1992)

11. Engel M, Hoffmann T, Wagner L, Wermann M, Heiser U, Kiefersauer R, Huber R, Bode W, Demuth HU, Brandstetter H. The crystal structure dipeptidyl peptidase IV (CD26) reveals its functional regulation and enzymatic mechanism. Proc. Natl. Acad. Sci. USA 100: 5063-5068 (2003) 
12. Conarello SL, Li Z, Ronan J, Roy RS, Zhu L, Jiang G, Liu F, Woods J, Zycband E, Moller DE, Thornberry NA, Zhang BB. Mice lacking dipeptidyl peptidase IV are protected against obesity and insulin resistance. Proc. Natl. Acad. Sci. USA 100: 6825-6830 (2003)

13. Rosenblum J, Kozarich JW. Prolyl peptidase: a serine protease subfamily with high potential for drug discovery. Current Opinion in Chemical Biology 7: 496-504 (2003)

14. Umezawa H, Aoyagi T, Ogawa K, Naganawa H, Hamada M, Takeuchi T. Diprotin A and B, inhibitor of dipeptidyl peptidase IV, produced by bacteria. J Antibiot 37: 422-425 (1984)

15. Reinhold D, Hemmer B, Gran B, Steinbrecher A, Brocke S, Kahne T, Wrenger S, Born I, Faust J, Neubert K, Martin R, Ansorge S. Dipeptidyl peptidase IV (CD26): role in T cell activation and autoimmune disease. Adv Exp Med Biol 477: 155-160 (2000)

16. Ahren B, Landin-Olsson M, Jansson PA, Sevensson M, Holmes D, Schweizer A. Inhibition of dipeptidyl peptidase-4 reduces glycemia, sustains insulin levels, and reduces glucagons levels in type 2 diabetes. J Clin Endocrinol Metab 89: 2078-2084 (2004)

17. Guan E, Wang J, Norcross MA. Amino-terminal processing of MIP-1 beta/CCL4 by CD26/dipeptidylpeptidase IV. J. Cell Biochem 92: 53-64 (2004)

18. Christopherson KW 2nd, Hangoc G, Broxmeyer HE. Cell surface peptidase CD26/dipeptidylpeptidase IV regulates CXCL12/stromal cell-derived factor- $\alpha$-mediated chemotaxis of human cord blood CD34+ progenitor cells. J Immunol 169: 7000-7008 (2002)

19. Broxmeyer HE, Kohli L, Kim CH, Lee Y, Mantel C, Cooper
S, Hangoc G, Shaheen M, Li X, Clapp DW. Stromal cell-derived factor-1/CXCL12 directly enhances survival/ antiapoptosis of myeloid progenitor cells through CXCR4 and $\mathrm{G}(\alpha) \mathrm{i}$ proteins and enhances engraftment of competitive, repopulating stem cells. J Leukoc Biol 73, 630-638 (2003)

20. Bristol LA, Bachovchin W, Laszlo T. Inhibition of CD26 enzyme activity with Pro-boropro stimulates rat granulocyte/ macrophage colony formation and thymocyte proliferation in vitro. Blood 85: 3602-3609 (1995)

21. Adams S, Miller GT, Jesson MI, Watanabe T, Jones B, Waller BP. PT-100, a small molecule dipeptidyl peptidase inhibitor, has potent antitumor effects and augments antibody-mediated cytotoxicity via a novel immune mechanism. Cancer Res 64: 5471-5480 (2004)

22. Jones B, Adams S, Miller GT, Jesson MI, Watanabe T, Waller BP. Hematopoietic stimulation by a dipeptidyl peptidase inhibitor reveals a novel regulatory mechanism and therapeutic treatment for blood cell deficiencies. Blood 102: 1641-1648 (2003)

23. Scanlan MJ, Raj BKM, Calvo B, Garin-chesa P, Sanzmoncasi MP, Healey JH, Old LJ, Rettig WJ. Molecular cloning of fibroblast activation protein $\alpha$, a member of the serine protease family selectively expressed in stromal fibroblasts of epithelial cancers. Proc Natl Acad Sci USA 91: 5657-5661 (1994)

24. Park JE, Lendr MC, Zimmermann RN, Garin-ches P, Old LJ, Rettig WJ. Fibroblast activation protein, a dual specificity serine protease expressed in relative human tumor stromal fibroblasts. J Biol Chem 274: 36505-36512 (1999) 